\title{
Las competencias disciplinares, educativas y socioafectivas en el perfil docente universitario
}

\author{
Yunuen I. Guzmán-Cedillo. Universidad Nacional Autónoma de México \\ Sandra Ivette Moreno Alcantara. Universidad del Valle de México \\ Natalia Lima-Villeda. Universidad Nacional Autónoma de México
}

Recepción: 2 de septiembre de 2015 | Revisión: 3 de septiembre de 2015 | Aceptación/Publicación: 7 de septiembre de 2015
Correspondencia: yunuenixchel@hotmail.com | sandra.moreno@uvmnet.edu |diana.limauvm@gmail.com
Citar: Guzmán-Cedillo, Yl., Moreno Alcantara, SI. y Lima-Villeda, N. (2015). Las competencias disciplinares, educativas y
socioafectivas en perfil docente universitario. ReiDoCrea, 4, 228-244. http://hdl.handle.net/10481/37268

Resumen: El objetivo del estudio es describir el proceso de construcción del modelo de competencias docentes en la Universidad del Valle de México (UVM). El tipo de diseño es descriptivo-transaccional. Las fases de desarrollo fueron documentación y adecuación empírica. La fase de documentación constó de la revisión de la literatura especializada, además del análisis de las instituciones de educación superior mejor posicionadas en los rankings mundiales. La adecuación empírica constó de la definición tanto conceptual como operacional, así también de la validación por 991 figuras académicas: estudiantes, docentes, coordinadores, directores y vicerrectores académicos. El resultado es un modelo de 19 competencias docentes divididas en tres grupos generales: Socioafectivas, Educativas y Disciplinares; competencias que son reconocidas por las figuras académicas (78\% de acuerdo en las Educativas, $100 \%$ en las Disciplinares y un $57 \%$ en las Socioafectivas motivacionales) así mismo, no hay diferencias entre áreas de conocimiento, lo que se determinó con la prueba de Kruskal-Wallis (ingenierías-hospitalidad $k=45.368, p<0.05$; saludnegocios $k=32.692, p<0.05$; hospitalidad-salud $k=59.041, p<0.01$ ). Se considera que el modelo de competencias docentes postula un perfil genérico del docente universitario. Para dar continuidad se sugiere la elaboración de instrumentos de evaluación del perfil para dar retroalimentación a la formación docente y, así, lograr la profesionalización en el marco de modelo. Así mismo es necesario determinar mecanismos para promover las competencias que no alcanzaron el $80 \%$ de acuerdo con miras a sensibilizar sobre la importancia de su presencia en la actividad docente en la Universidad.

Palabras clave: Competencias del docente | Educación Superior

Disciplinary, Educational and Socio-Affective Competencies in the Profile of University Faculty

Abstract: The objective of this research is to describe the elaboration process of model teacher's competencies in college at UVM (Universidad del Valle de México). The study design is descriptive. The research was made in two phases: documentation and empirical adaptation. In the first phase the literature and the best practices in universities in world education's rankings were analyzed. In second phase an empirical adaptation was made. There were 991 participants from the community of the university. The model has 19 competences divided in three general groups: motivational, educative and disciplinary competences all of them are recognized as important by university community ( $78 \%$ in educative, $100 \%$ in disciplinary and $57 \%$ of agreement in motivational competences). Also the results show that there were not significance differences between areas of knowledge in Kruskal-Wallis test (engineering-hospitality $k=45.368, p<0.05$; health-business administration $k=32.692, p<0.05$; hospitality-health $k=59.041, p<0.01$ ). Because of that, the model is a generic teacher's profile competencies at UVM. The next steps will search to make assessment instruments to evaluate the teacher's profile in order to give a way to promote the college teacher professionalization.

Keywords: Teacher qualifications | Higher Education

\section{Introducción}

La aproximación educativa basada en competencias ha mostrado una diferenciación del desarrollo de las habilidades que los estudiantes logran en su formación (Murzakhanovna, Meirbekovich, Erkinbekovich, Pernebekovna, Koptleuovich y Kyrgyzbaevna, 2013).

Actualmente uno de los retos para las Instituciones de Educación Superior (IES) es ofrecer servicios de calidad. Uno de ellos está encaminado en brindar a los estudiantes espacios de desarrollo de capacidades profesionales, necesarias para una inserción exitosa en el campo laboral. Por ello uno de los factores que inciden en el logro de la calidad educativa, son sin lugar a dudas las competencias docentes (Pereyra, 2014, Witte, 2015). 
La calidad del docente es un factor muy importante que influye en el logro académico estudiantil (Hilton, Flores y Niklasson, 2013; en Witte, 2015), por ello debe ser una prioridad en las instituciones educativas que involucren el desarrollo de perfiles o de un estándar de calidad que haga pensar a los docentes en diferentes formas sobre su trabajo, el salón de clases, las actividades de aprendizaje y la identidad profesional.

El docente elabora un marco normativo cultural de valores y metas que guían su práctica educativa, dependiendo de las experiencias, la política institucional y su contexto (Webel y Patt, 2015).

El término competencias docentes refiere al conjunto de conocimientos, habilidades y actitudes del docente, que se relacionan entre sí durante el proceso educativo de tal forma que culminan con el desempeño exitoso tanto de las actividades como de las funciones de la práctica docente. A dicha articulación se adhiere el dominio de una disciplina particular que interviene en la planificación de los procesos de enseñanza aprendizaje que sean acordes a los contenidos del campo de conocimiento planteados en un plan o programa de estudio en el caso de la universidad (Guzmán-Cedillo y Lima-Villeda, 2014; Mas Torelló, 2012).

Bajo la perspectiva de competencias, se reconoce la importancia de la capacidad del docente para estimular el interés de sus estudiantes por aprender nuevos conocimientos, preocuparse por ellos al mostrar una actitud positiva al estudiantado, además de manifestar entusiasmo por el contenido de la enseñanza que promueve, debe motivar a los alumnos para ser responsables de su proceso de aprendizaje, a la vez que fomenta actitudes y valores positivos hacia la profesión, por lo que los contenidos abordados le permiten explicar situaciones de la vida cotidiana.

Por ejemplo, los aspectos motivacionales y sociafectivos, juegan un papel importante en lo que enseñan los docentes. Se reconoce que las creencias, los valores, la motivación y las habilidades de autoeficacia del docente se relacionan con la disposición a la adaptación de sus estrategias de enseñanza. Es decir, Hachfeld, Hahn, Scroeder, Anders y Kunter (2015) ubicaron que el tipo de creencias respecto a la diversidad cultural de los docentes se relacionan con la forma en que modulan las estrategias de enseñanza que implementan cuando tienen estudiantes pertenecientes a minorías.

Por otro lado, Dolby y Lister (2015) encontraron que los profesionales no tienen presente el área potencial de formar o "educar" a otros durante su formación, es decir, minimizan el rol educativo que el profesional puede tener. En el caso de su investigación con Médicos Veterinarios, existe una minimización de la parte pedagógica frente al desarrollo del conocimiento disciplinar. Encuentran que los estudiantes se enfocan mucho al campo de cuidado de los animales (componente disciplinar), y que el rol educativo se centra en dar los conocimientos sobre cómo cuidar a los animales. Así también, consideran que esto puede deberse a que curricularmente no se recupera ese componente educativo en la formación y por ende, el estudiante no lo ve como posible campo de desarrollo profesional.

Diferentes estudios (Flores-Hernández, Martínez-González, Sánchez-Mendiola, García-Cabrero y Reidl, 2010; Rueda, 2006; Guzmán y Marín 2011) subrayan la importancia de las capacidades docentes en diferentes áreas relacionadas a los procesos de enseñanza aprendizaje, las relaciones socioafectivas y motivacionales tanto con los compañeros docentes como con los estudiantes, además de los aspectos específicos de la disciplina que enseña, elementos que concatenan el sentido ético de cada profesión. 


\section{Los rankings universitarios}

En un contexto donde el mercado es el eje de desarrollo, la economía se basa en el conocimiento para satisfacer necesidades sociales, la educación es una necesidad que los rankings han aprovechado, ya que su objetivo es evidenciar la posición que las instituciones educativas ostentan en la oferta de formación a nivel nacional e internacional. Estas posiciones se determinan a través de una selección de indicadores, con los que se pondera y asigna puntaje a las instituciones que se evalúan. En consecuencia los resultados que las IES obtienen en los rankings son importantes para la elección que pueden realizar los aspirantes y el reconocimiento como una institución de calidad. En lo que respecta a los docentes, los rankings refieren elementos relacionados de forma directa con esta figura, estos elementos refieren a las estrategias educativas implementadas, la reputación entre los estudiantes, el intercambio con otras instituciones así como el reconocimiento entre pares y su producción científica (Academic Ranking of World Universities, 2014; QSIntelligence Unit, 2014; Times Higher Education, 2015).

\section{Contexto en la Universidad del Valle de México (UVM)}

La UVM es una institución educativa de carácter privado, dedicada a la formación media superior y superior. En el caso de la formación superior se enfoca a formar a personas de manera integral, competentes profesionalmente a nivel nacional e internacional, acorde a las necesidades sociales. Dentro de su oferta educativa profesional atiende a dos perfiles de ingreso diferentes, uno refiere a los estudiantes de tiempo completo y los estudiantes que combinan el estudio con el trabajo o son graduados que desean continuar su formación en un posgrado. En ese sentido los docentes en ambas modalidades de formación tienen un perfil general que contempla habilidades para el ejercicio profesional y para la docencia. Es decir, se busca que además de tener un dominio sobre el contenido disciplinar también tengan conocimientos pedagógicos que faciliten su ejercicio docente. Al respecto, en la UVM se ha buscado promover el desarrollo de competencias docentes que repercutan en la mejora de los procesos educativos, por ello se considera necesario generar una investigación educativa cuyos resultados permitan la conformación de un modelo que enmarque el perfil docente de la institución. Por ello, se considera que un proceso sistemático de recopilación de información y validación de la propuesta por parte de diferentes figuras académicas ayuda al desarrollo de una visión compartida de las competencias deseables en la institución. Derivado de ello, la pregunta de investigación planteada refiere a ¿Cuáles son las competencias que deben integrar el modelo de competencias docentes que conforman el perfil del profesor en la UVM?

Con base en lo anterior el objetivo de este estudio es describir el proceso de elaboración del modelo de las competencias docentes que conforman el perfil del profesor en la Universidad del Valle de México desde la perspectiva de las diferentes figuras académicas.

\section{Método}

\section{Participantes}

En este estudio hubo 991 involucrados con diferentes roles y funciones:

- Cinco investigadores educativos, con formación profesional relacionada con la educación y el trabajo desde el enfoque por competencias. Tres de ellos con experiencia docente universitaria. 
- Un coordinador de formación docente.

- Dos lectores. Una Licenciada en Comunicaciones con Maestría en Pedagogía que se desempeña como diseñadora curricular en UVM y un Licenciado en Letras Hispánicas, quienes revisaron la redacción de cada uno de los grupos de las competencias y de las competencias.

- Treinta y siete estudiantes de tiempo completo inscritos en la Universidad del Valle de México pertenecientes a diferentes semestres y carreras (Arquitectura, Ciencias de la Comunicación, Contaduría, Derecho, Enfermería, Gastronomía, Ingeniería en sistemas, Ingeniería industrial, Ingeniería en Tecnología Interactiva en Animación Digital, Mercadotecnia, Mercadotecnia Deportiva, Médico Veterinario Zootecnista, Nutrición y Psicología).

- Novecientas cuarenta y seis figuras académicas de la Universidad (tabla 1).

\begin{tabular}{|lc|}
\hline \multicolumn{2}{|c|}{ Tabla 1 } \\
Figuras académicas que participaron en la validación del perfil \\
\hline Figuras académicas & Número de figuras \\
\hline Docentes modalidad de tiempo completo & 633 \\
Vicerrectores & 8 \\
Docentes modalidad tiempo parcial y posgrado & 53 \\
Coordinadores y directores de la modalidad de tiempo completo & 234 \\
Coordinadores y directores de la modalidad de tiempo parcial & 8 \\
Coordinadores y directores de posgrado & 3 \\
Coordinadores y directores de prepa & 7 \\
\hline
\end{tabular}

\section{Instrumentos}

Dos escalas de evaluación y dos de autoevaluación docente pertenecientes al Sistema Integral de Evaluación del Desempeño Docente (SIEDD) de la UVM. Dos de ellas corresponden a la opinión estudiantil en las modalidades de estudiante de tiempo completo además de adulto trabajador. Las cuatro contienen dos reactivos con respuestas abiertas, además de ser reactivos tipo Likert entre 18 y 20 reactivos en cada una. Las escalas fueron validadas por 667 docentes de la institución. Al ser piloteadas mostraron una confiabilidad buena: $\alpha 0.80$, medida a través del alpha de Cronbach (Pereyra, Lima-Villeda y Armenta, 2014).

Los reactivos con respuesta abierta para los estudiantes son: "Menciona dos aspectos positivos de tu profesor" y "Menciona dos aspectos negativos de tu profesor". Los reactivos con respuesta abierta para los docentes son: "Mencione dos aspectos positivos de su práctica docente" y "Mencione dos aspectos negativos de su práctica docente".

Encuesta NPS o Net Promoter Score (Reichheld, 2006), índice que a través de un cuestionario permite identificar la relación existente entre la satisfacción de los estudiantes y las perspectivas de crecimiento de la institución con el fin de incrementar la lealtad de los estudiantes, con una pregunta ellos responden la satisfacción lograda con relación a la institución. La respuesta se asocia con una escala de 1 a 10 . Con base en esta respuesta, se clasifican de la siguiente forma: calificaciones entre 1 y 6 corresponde a Detractores, calificaciones entre 7 y 8 corresponde a Neutros o Pasivos y calificaciones entre 9 y 10 corresponde a Promotores.

Seis cuestionarios desarrollados en la investigación alojados en un sitio de internet, cuyo propósito fue que los participantes validaran las competencias del modelo en una escala nominal con tres posibilidades de respuesta: 1.Necesarias, 2 Útiles pero no 
necesarias o 3 No necesarias. Los cuestionarios constan de tres secciones: la primera para recabar datos de identificación, la segunda de presentación, instrucciones y definiciones de las competencias del modelo, además de la tercera con 19 preguntas para clasificar las competencias. La diferencia entre los cuestionarios radica en la sección para recabar datos de identificación.

Materiales: laptop y tableta electrónica.

\section{Unidades de análisis}

- 10 Sitios de formación docente de las instituciones mejor posicionadas en los rankings internacionales

- 1338 comentarios retomados de las respuestas abiertas de las escalas de evaluación docente del SIEDD, las cuales hacen alusión a aspectos positivos y aspectos negativos del docente.

- 3775 respuestas a la encuesta NPS.

- 938 respuestas de las figuras académicas a los cuestionarios.

\section{Procedimiento}

La propuesta se ha construido por medio de dos fases: documentación y adecuación empírica, a su vez, entre ambas fases, la construcción del modelo se compone de siete pasos.

\section{Fase de documentación}

\section{Análisis de rankings}

Identificación de competencias docentes en las universidades posicionadas en los primeros lugares de rankings educativos. Se identificaron las competencias docentes más frecuentemente fomentadas en la formación docente de las universidades ubicadas en las primeras posiciones de tres rankings internacionales. (Guzmán-Cedillo y Lima-Villeda, 2013)

2. Revisión de literatura sobre competencias docentes. En la literatura educativa se reconocieron propuestas sobre el tema de Educación superior, su definición e indicadores de medida para generar una primera caracterización.

\section{Fase de adecuación empírica}

\section{Comprobación en SIEDD}

Se categorizaron a través de la técnica de análisis de contenido las respuestas abiertas de los estudiantes y docentes con respecto a las competencias a las que hacían alusión. Dicho análisis consistió en la revisión de cada una de las respuestas de los estudiantes y docentes, se etiquetaron a partir de si hacían referencia a uno de los tres grupos de competencias: Educativas, Socioafectivas o Disciplinares.

\section{Definición de competencias}

Con las respuestas codificadas se inició una fase de conceptualización de las competencias. Este trabajo lo llevaron a cabo dos investigadoras educativas y un coordinador de formación docente. Se retomaron las definiciones localizadas en la 
literatura educativa, de tal forma que al hacer un comparativo entre los elementos comunes se definieron cada uno de los grupos de competencias. Las definiciones de cada grupo de competencias, resultado de este trabajo fueron revisadas por dos lectores expertos en lengua y comunicación educativa.

Ulteriormente, las dos investigadoras educativas y el coordinador de formación docente revisaron conjuntamente cada competencia, se señalaron los elementos constitutivos de las competencias, retomándolos para su conjunción y descripción. Se comparó la definición con los extractos codificados en el paso 3 para corroborar que se incluyeron los elementos que mencionaron tanto docentes como estudiantes. Durante este proceso se eliminaron competencias debido a que algunas se traslapaban entre ellas.

Posteriormente, tres investigadores educativos revisaron si las competencias estaban clasificadas en el grupo que les correspondía, según la definición del grupo de competencias.

\section{Revisión de comentarios NPS}

Las definiciones realizadas en la fase anterior conformaron una rejilla de análisis, con la cual se categorizaron en las competencias docentes a las respuestas de la encuesta NPS, por medio de la técnica de análisis de contenido, de manera independiente por tres investigadoras educativas hasta alcanzar más del $80 \%$ de acuerdo entre ellas. Fueron cinco momentos de revisión, en cada momento se trabajó con una muestra de 10 respuestas de la encuesta NPS.

\section{Piloteo}

Para revisar que las definiciones de las competencias eran comprendidas por la comunidad, se llevó a cabo un piloteo con 37 estudiantes de tiempo completo y ocho docentes de la misma modalidad. Se utilizaron dos cuestionarios, uno para docentes y otro para estudiantes en la zona de esparcimiento dentro de la universidad, la aplicación fue por medio de una laptop y una tableta electrónica. Los datos se recabaron en una base de datos virtual, generada en el momento en que se concluyó el cuestionario. En la validación, tanto los estudiantes como los docentes mencionaron si las competencias eran Necesarias o Útiles pero no necesarias o No necesarias.

\section{Validación del modelo con las figuras académicas}

Las competencias docentes fueron validadas en términos de si las diferentes figuras académicas las consideraban en una de las siguientes posibilidades para la enseñanza en la institución: Necesarias, Útiles pero no necesarias o No necesarias. Se diseñó y envió de forma electrónica los cuestionarios, de tal forma que los datos se recabaron en una base virtual. Por medio de la Coordinación Institucional de Formación Docente de la Universidad (UVM) fueron enviados los cuestionarios a las figuras académicas.

\section{Resultados}

A continuación los datos obtenidos se presentan por cada una de las fases y pasos realizados:

Fase de documentación 


\section{Análisis de rankings}

Se encontraron 25 competencias docentes referidas en los sitios de las instituciones. En la figura 1 se puede observar que las competencias más frecuentes son: dinamismo y entusiasmo, adaptación o pertinencia de las prácticas a partir de la actividad, comunicación con los estudiantes así como la promoción de un vínculo entre estudiantes y profesores. Las instituciones que más competencias mencionan es Berkeley con 13 competencias y California Institute of Technology (Caltech) con 11 competencias. En la figura 1 Berkeley se muestra de color gris y Caltech con color azul oscuro.

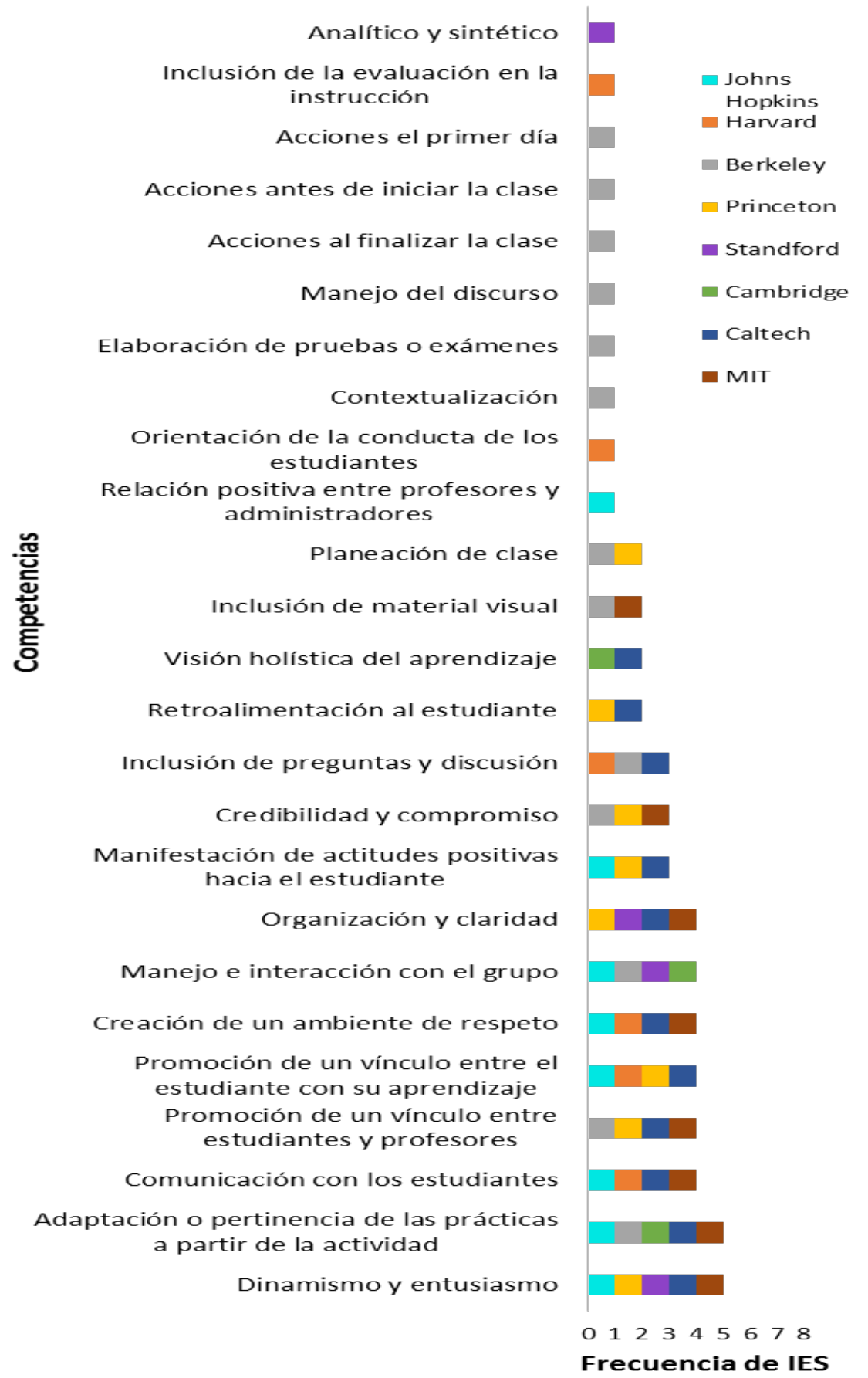

Figura 1. Competencias docentes más frecuentes en los sitios web de las IES posicionadas en los primeros lugares de tres rankings internacionales. Fuente: elaboración propia 
2. Revisión de literatura sobre competencias docentes

Al realizar el análisis de las propuestas en la literatura educativa, se encontró que las capacidades docentes están relacionadas con tres aspectos de forma preponderante: las relaciones socioafectivas motivacionales, el proceso de enseñanza aprendizaje así como los aspectos específicos de la disciplina que se enseña.

Cabe señalar que se compararon las competencias con las encontradas en la literatura, lo cual, no sólo permitió su agrupación, sino que también algunas competencias fueron fusionadas como Planeación de clase, Acciones antes y al finalizar la clase. Aunado a ello, se agregaron otras competencias, tales como Recursos didácticos, Búsqueda de retroalimentación, Material didáctico, Formación y experiencia docente y Técnicas didácticas, teniendo en total 28 competencias, en esta primera fase de desarrollo del modelo.

\section{Comprobación en SIEDD}

Las respuestas abiertas de los cuestionarios del SIEDD dieron paso al análisis de 1338 comentarios, que reflejan la presencia de las competencias docentes desde la percepción de los estudiantes y de los docentes, ver figura 2 . Se encontró que la mayor parte de los comentarios refirieron a competencias Educativas (47\%), posteriormente las Disciplinares $(24 \%)$ y por último las competencias Socioafectivas motivacionales $(24 \%)$

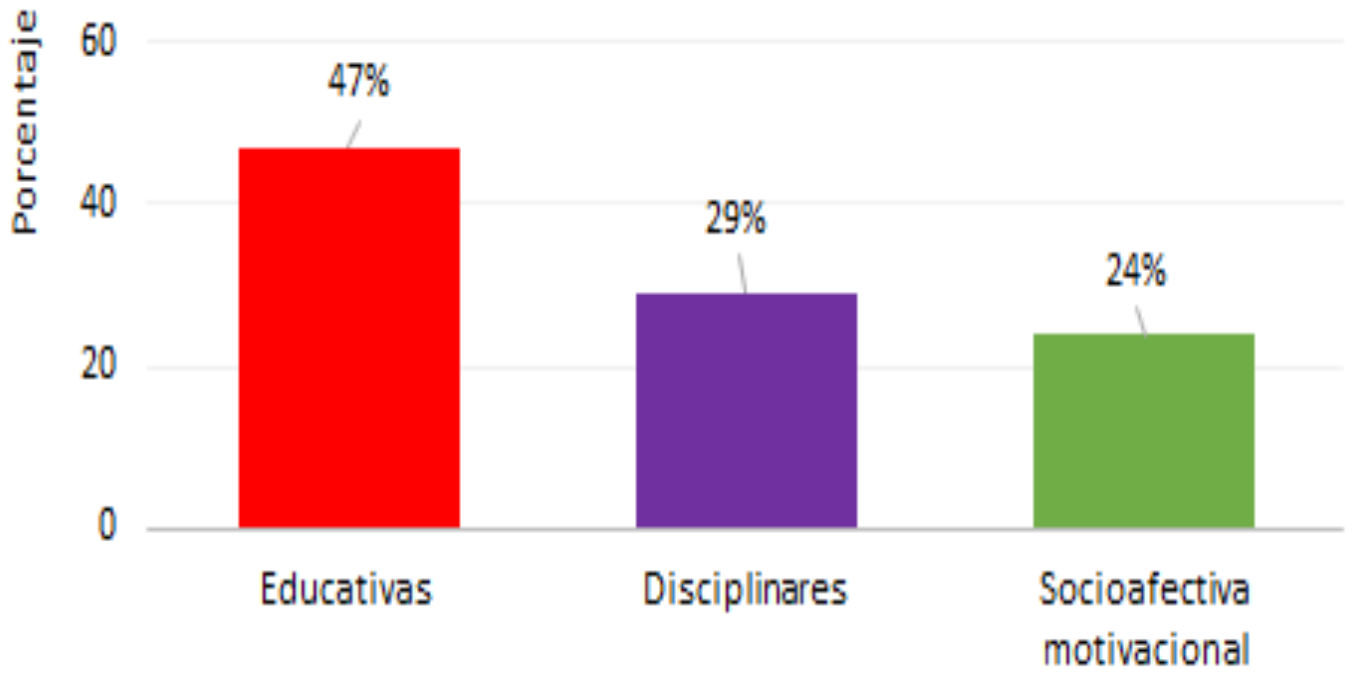

Competencias

Figura 2. Muestra la presencia de las competencias docentes en los comentarios de estudiantes y docentes en las preguntas abiertas de los cuestionarios de evaluación docente implementados por el SIEDD. Fuente: elaboración propia 
Algunos comentarios de los estudiantes y los docentes que ejemplifican cada grupo de competencias se muestran en la tabla 2.

\begin{tabular}{|c|c|c|}
\hline \multicolumn{3}{|c|}{$\begin{array}{c}\text { Tabla } 2 \\
\text { Comentarios a los reactivos con respuesta abierta }\end{array}$} \\
\hline Competencia & Comentarios de estudiantes & Comentarios de docentes \\
\hline Educativa & $\begin{array}{l}\text { "Las actividades fuera del aula, programadas } \\
\text { por la maestra, permiten que el alumno } \\
\text { reflexione, investigue y profundice los } \\
\text { contenidos de la materia." } \\
\text { "Se detiene si algún alumno no comprende el } \\
\text { tema" }\end{array}$ & "Pedir retroalimentación a los alumnos" \\
\hline Disciplinar & $\begin{array}{l}\text { "Domina la materia en más del } 100 \% " \\
\text { "Es un profesor muy preparado, con mucho } \\
\text { conocimiento sobre el tema" } \\
\text { "Tiene experiencia profesional en el tema" }\end{array}$ & $\begin{array}{l}\text { "Ejemplos de hoy en día" } \\
\text { "Aterrizar a tiempo los conceptos que se revisan } \\
\text { en clase" }\end{array}$ \\
\hline $\begin{array}{l}\text { Socioafectiva } \\
\text { motivacional }\end{array}$ & $\begin{array}{l}\text { "Buen colaboradora, apoya a los alumnos, } \\
\text { atento al proporcionarnos la información } \\
\text { cuando la requerimos" } \\
\text { "La disponibilidad para aclararnos dudas fuera } \\
\text { de horario de clase" }\end{array}$ & $\begin{array}{l}\text { "Contribuir en el nuevo modelo educativo por } \\
\text { competencias, para que los estudiantes puedan } \\
\text { ser competitivos y logren insertarse en el mundo } \\
\text { globalizado" } \\
\text { "Incluir en todas las cátedras la filosofía de } \\
\text { nuestra universidad" }\end{array}$ \\
\hline
\end{tabular}

\section{Definición de competencias}

La revisión de literatura y los extractos de los comentarios analizados en las etapas anteriores, dotaron de indicadores para reconocer y definir los tres grupos de competencias docentes: Educativas, Disciplinares y Socioafectivas-motivacionales.

A las competencias Educativas se les comprende como a todas aquellas capacidades del docente para organizar situaciones de enseñanza aprendizaje al planear, diseñar, implementar y evaluar el logro de las metas, identificando tanto la necesidad de formación que plantea el programa como las características del estudiante, elementos que serán guiadas en este proceso. Además de la inquietud por parte del docente de leer artículos, libros o participar en eventos académicos sobre educación y didáctica.

Las competencias Disciplinares refieren a aquellas capacidades del docente en torno a un campo de conocimiento o disciplina profesional, que corresponden a una serie de postulados, conceptos fenómenos, soluciones, métodos y leyes. Además de la manifestación por parte del docente de leer artículos, libros o la participación en eventos académicos sobre cómo se enseña su disciplina.

Finalmente las competencias Socioafectivas-motivacionales son las capacidades del docente para relacionarse con sus estudiantes y promover relaciones positivas entre ellos, apoyándose en el diálogo que estimula la comunicación, la retroalimentación positiva, la empatía, la motivación, la calidad humana y la transmisión del entusiasmo por aprender. Así mismo involucra la capacidad de resolución de conflictos así como la promoción de la apreciación de sí mismo y la autonomía; además de guiar al estudiante al logro de las expectativas académicas. En resumen, dichas capacidades se relacionan con aquellas actitudes que manifiestan el interés y la disponibilidad para atender a los estudiantes. 
La definición de las competencias a su vez permitió reconocer de forma precisa las competencias que conforman cada uno de los tres grandes grupos, de tal forma que, las 28 competencias anteriormente ubicadas, se concentraron en 21 competencias, dado que algunas al momento de describirlas se traslapaban.

\section{Revisión de comentarios NPS}

La encuesta NPS proporcionó 3775 comentarios de estudiantes sobre docentes, comentarios examinados por medio de un análisis de contenido.

Las tres competencias estuvieron presentes, sin embargo, cabe señalar que emergió una categoría que no correspondía a las competencias docentes, dicha categoría fue llamada Extra. Los comentarios agrupados en esa sección, estuvieron enfocados a comentarios en los que su contenido manifiesto no se relacionaba con las competencias docentes. En la figura 3 se muestra el porcentaje de presencia de las competencias en los comentarios.

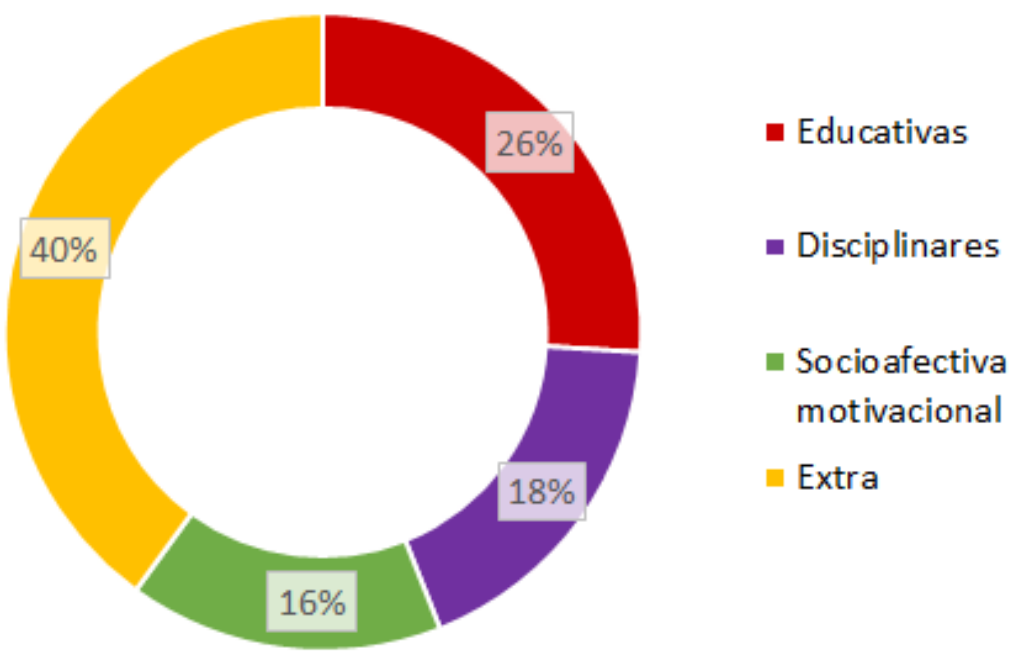

Figura 3. Porcentaje de presencia en los comentarios de estudiantes en la encuesta NPS. Fuente: elaboración propia

Específicamente, la presencia de las competencias educativas estuvo concentrada en Formación y Experiencia Docente, Técnicas Didácticas y Planeación de la Clase, como se muestra en la tabla 3.

\begin{tabular}{|lc|}
\hline & $\begin{array}{c}\text { Tabla } 3 \\
\text { Presencia de competencias educativas }\end{array}$ \\
\hline Competencias Educativas & Porcentaje de presencia en los comentarios \\
\hline Formación y Experiencia Docente & 32.0 \\
Técnicas Didácticas & 21.0 \\
Planeación de la Clase & 20.5 \\
\hline Nota. Muestra la presencia de las competencias educativas en los comentarios de los estudiantes que alcanzaron más \\
del 10\% de presencia en los comentarios. El total de los comentarios relacionados a las competencias educativas \\
fueron 1001. \\
\hline
\end{tabular}


Las competencias disciplinares tuvieron un alto porcentaje de presencia cada una. La competencia Dominio de la Asignatura tuvo la mayor presencia en los comentarios, tal como se muestra en la tabla 4.

\begin{tabular}{|lc|}
\hline \multicolumn{2}{|c|}{$\begin{array}{c}\text { Tabla 4 } \\
\text { Presencia de competencias disciplinares }\end{array}$} \\
\hline Competencias Disciplinares & Porcentaje de presencia en los comentarios \\
\hline Dominio de la Asignatura & 48.5 \\
Formación y Experiencia Profesional & 34.5 \\
$\begin{array}{l}\text { Pertinencia de las Actividades de Aprendizaje de Acuerdo a la } \\
\text { Profesión }\end{array}$ & 17.0 \\
\hline $\begin{array}{l}\text { Nota. Muestra la presencia de las competencias disciplinares en los comentarios de los estudiantes. El total de los comentarios relacionados a las } \\
\text { competencias educativas fueron 685. }\end{array}$ \\
\hline
\end{tabular}

Las competencias Socioafectivas motivacionales con mayor presencia fueron Manifestación de Valores y Actitudes Positivas hacia el Estudiante así como Promoción de un Vínculo con sus Estudiantes (véase tabla 5).

\begin{tabular}{|l|c|}
\hline \multicolumn{2}{|c|}{ Tabla 5 } \\
\hline \multicolumn{2}{|c|}{ Presencia de competencias Socioafectivas motivacionales } \\
\hline Competencias Socioafectivas motivacionales & Porcentaje de presencia en los comentarios \\
\hline Manifestación de Valores y Actitudes Positivas hacia el Estudiante & 48.0 \\
\hline Promoción de un Vínculo con sus Estudiantes & 26.5 \\
\hline Promoción de un Vínculo entre el Estudiante con su Aprendizaje & 14.0 \\
\hline $\begin{array}{l}\text { Nota. Muestra la presencia de las competencias educativas en los comentarios de los estudiantes que alcanzaron más del 10\% de presencia en } \\
\text { los comentarios. El total de los comentarios relacionados a las competencias educativas fueron 597 }\end{array}$ \\
\hline
\end{tabular}

\section{Piloteo}

Ninguna de las 21 competencias fue considerada No necesaria por parte de los docentes y la mayor parte es considerada Necesaria. Al comparar los resultados entre los tres grupos de competencias (véase figura 4), se encontró que las competencias Socioafectivas motivacionales tuvo menor porcentaje de competencias consideradas Necesarias, las restantes son catalogadas Útiles pero no necesarias, las cuales son Ajuste durante la clase y Construcción de un vínculo con sus estudiantes.

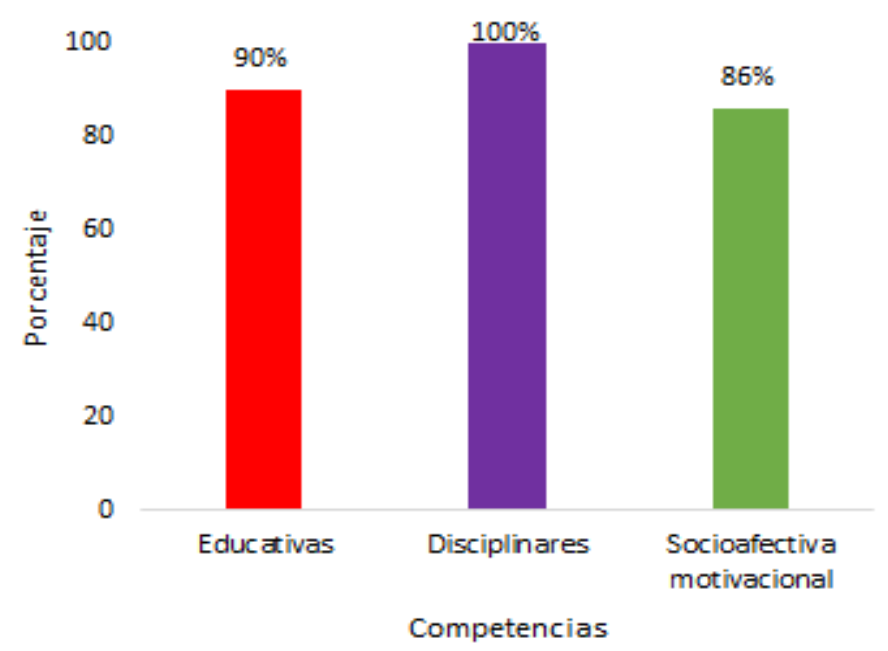

Figura 4. Muestra el porcentaje de competencias consideradas Necesarias desde la valoración de los docentes. Fuente: elaboración propia. 
Los resultados de los estudiantes no difieren de los resultados de los docentes. Como se muestra en la figura 5, la mayor parte de las competencias son consideradas Necesarias. Al realizar el comparativo entre los tres grupos de competencias, se encontró que las disciplinares tuvieron un $100 \%$ de acuerdo entre los estudiantes al categorizarlas Necesarias, el mismo resultado de la validación de los docentes.

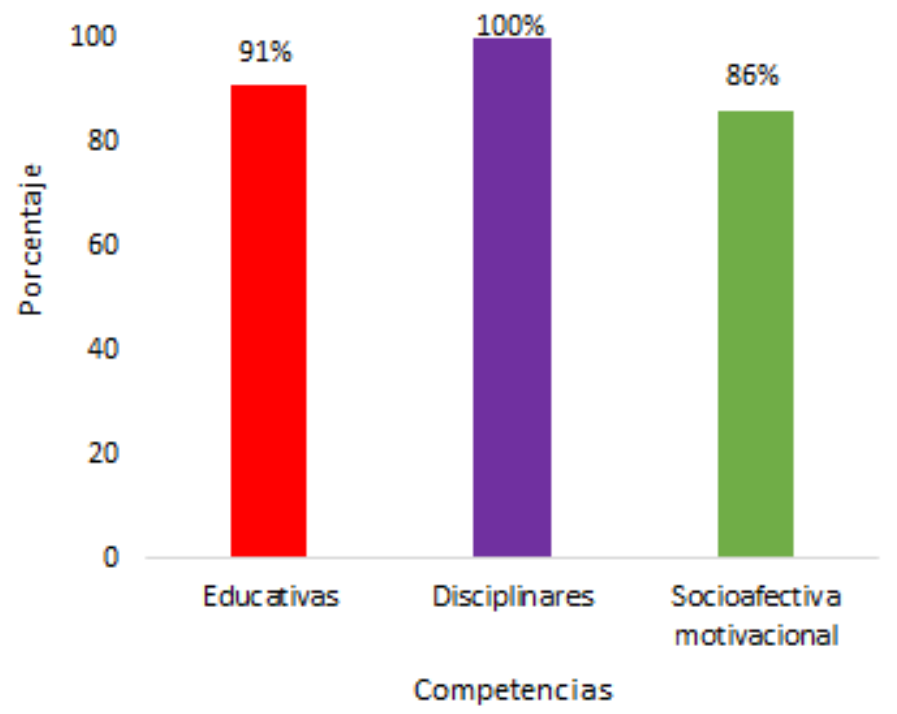

Figura 5. Muestra el porcentaje de las competencias consideradas Necesarias desde la valoración de los estudiantes. Fuente: elaboración propia.

\section{Validación del modelo con las figuras académicas}

A partir de la validación de los docentes de la modalidad de tiempo completo, Vicerrectores, Directores y Coordinadores se encontró que sólo un poco más de la mitad de las competencias Socioafectivas motivacionales son Necesarias (57\%) y más de la mitad de las competencias Educativas (78\%) también son Necesarias. Caso contrario a las competencias Disciplinares, que el $100 \%$ de las competencias lo son (véase figura 6). Las competencias que son validadas como Útiles pero no necesarias fueron: Ajuste durante la clase, Uso de recursos y materiales didácticos, Orientación de la conducta de los estudiantes, Relación positiva entre los diferentes actores de la comunidad escolar y Promoción de un vínculo con sus estudiantes. Estas son competencias que no alcanzaron el $80 \%$ de acuerdo entre docentes.

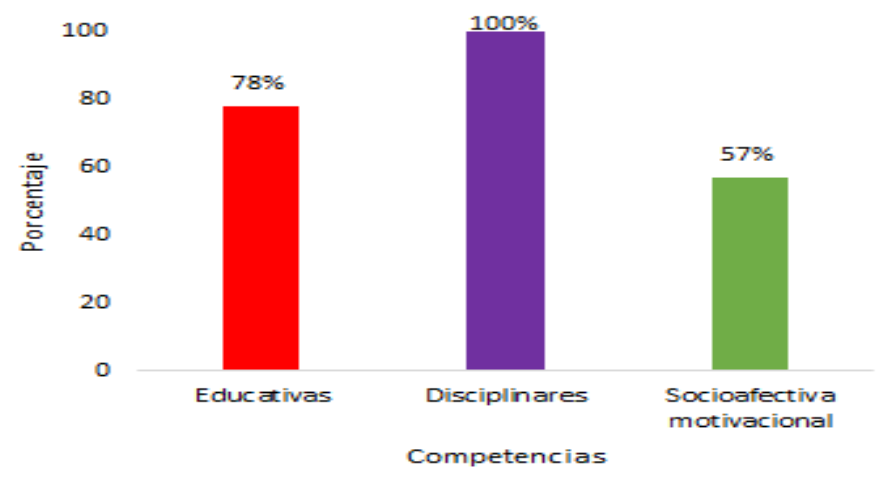

Figura 6. Muestra el porcentaje de las competencias Educativas, Disciplinares y Socioafectivas motivacionales considerandas Necesarias por los docentes de la modalidad de tiempo completo, Vicerrectores, Directores y Coordinadores. Fuente: elaboración propia. 
El 74\% de las competencias son consideradas Necesarias (véase figura 7).

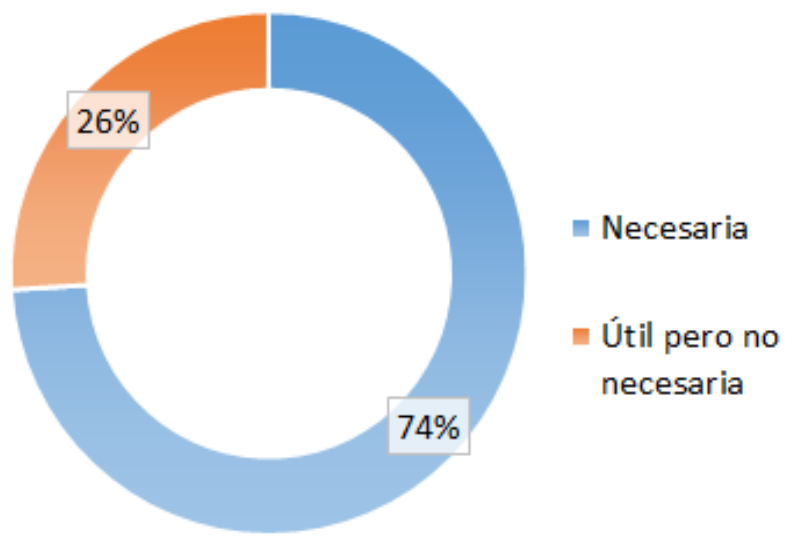

Figura 7. Se muestra el porcentaje de competencias validadas Necesarias y Útiles pero no necesarias según los docentes de la modalidad de tiempo completo, Vicerrectores, Directores y Coordinadores. Fuente: elaboración propia.

Los resultados de la prueba Kruskal-Wallis fue aplicada para reconocer diferencias entre áreas de conocimiento y se encontró que no existen diferencias entre las mismas. La única competencia que mostró diferencias significativas entre las áreas de conocimiento fue Manejo de la interacción con el grupo (véase figura 8). Se encontró que las diferencias fueron entre las Ingenierías y Hospitalidad, Turismo además de Gastronomía $(k=45.368, p<0.05)$; las carreras de Salud y de Negocios $(k=32.692$, $p<0.05)$ y entre Salud y Hospitalidad, Turismo y Gastronomía $(k=59.041, p<0.01)$.

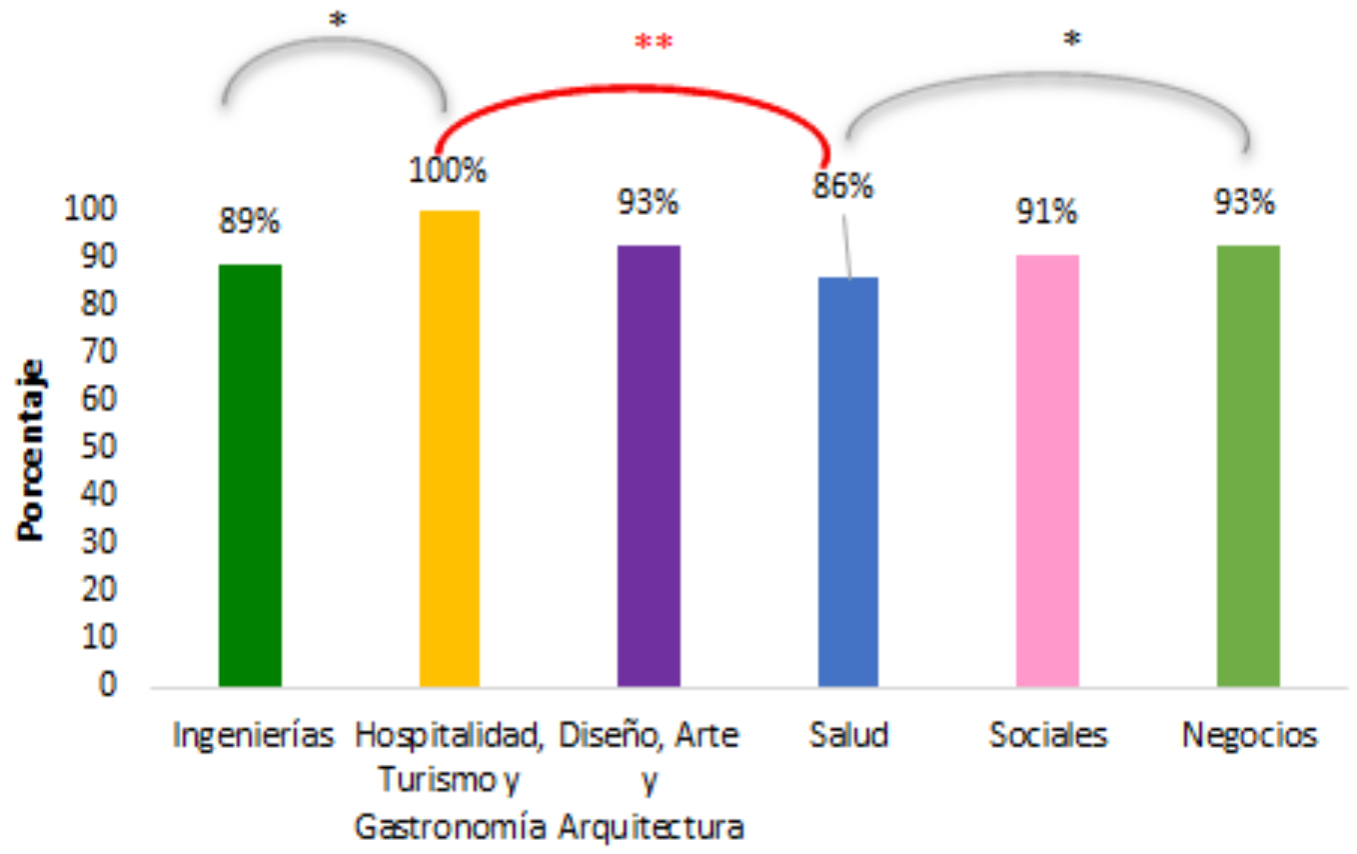

\section{Áreas de formación}

Figura 8. Muestra el porcentaje de participantes que identificaron la competencia Manejo de la interacción con el grupo como Necesaria así como las diferencias significativas. Un solo asterisco $\left(^{*}\right)$ muestra que se encontraron diferencias al nivel 0.05 ; dos asteriscos $\left(^{* *}\right)$ muestra que se encontraron diferencias al nivel 0.001. Fuente: elaboración propia. 
Respecto de la validación de los docentes de la modalidad de tiempo parcial, se encontró que el $63 \%$ de las competencias son validadas como Necesarias (véase figura 9), de las cuales el $67 \%$ son Educativas, $43 \%$ Socioafectivas-motivacionales y el $100 \%$ son Disciplinares, como se muestra en la figura 10.

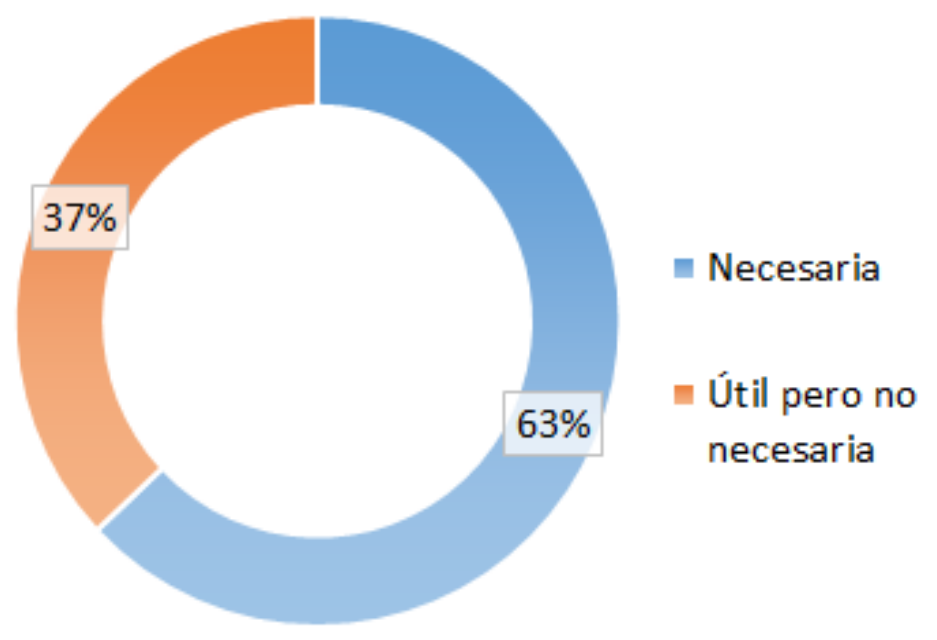

Figura 9. Muestra el porcentaje de competencias Necesarias y Útiles pero no necesarias por los docentes de la modalidad Adulto Trabajador. Fuente: elaboración propia.

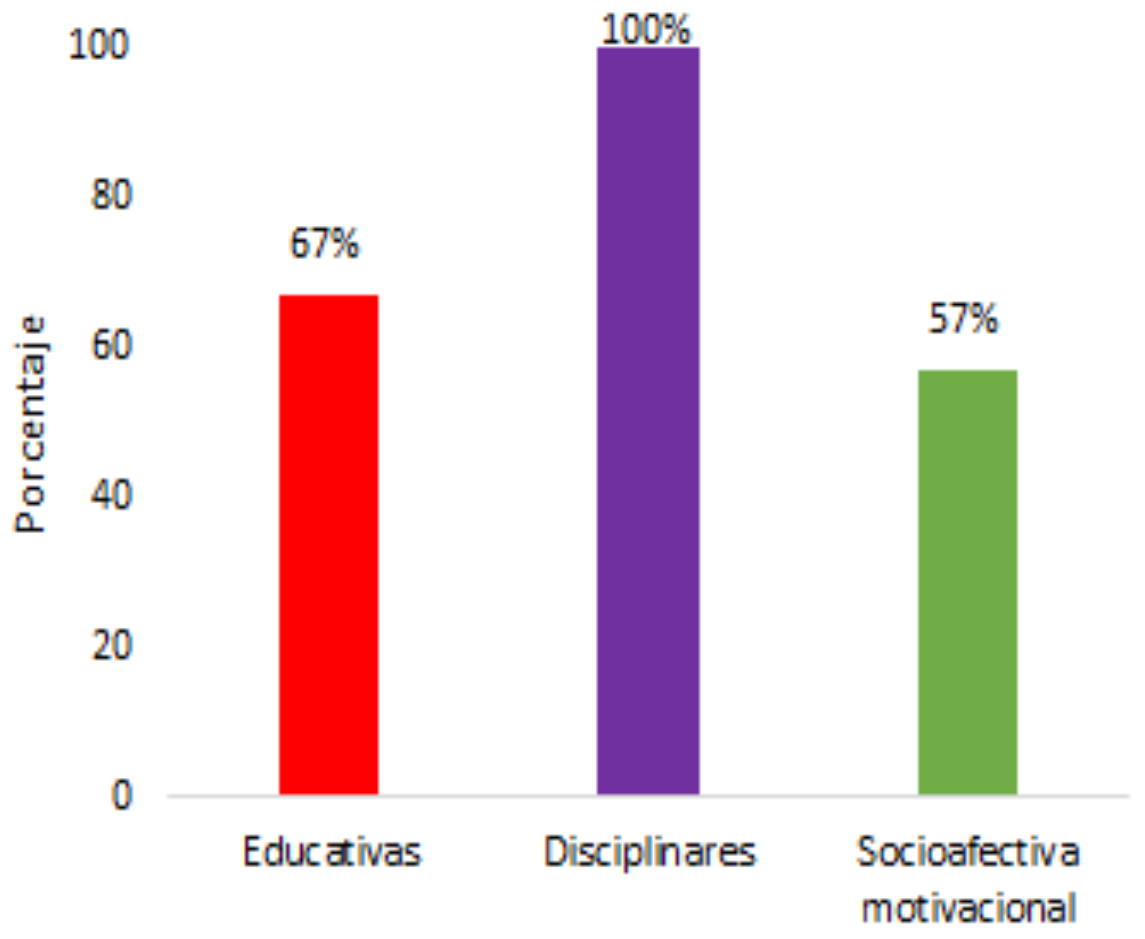

Competencias

Figura 10. Muestra el porcentaje de competencias Necesarias y Útiles pero no necesarias al validarlas por docentes de la modalidad de Adulto Trabajador. Fuente: elaboración propia. 
En la figura 11 se muestra el modelo de competencias, señalando con $(v)$ las competencias validadas como necesarias por las figuras académicas, de las cuales, son seis competencias Educativas: organización y claridad en el discurso, planeación de la clase, evaluación de la clase, búsqueda de retroalimentación, formación y experiencia docente y técnicas didácticas; son las tres competencias Disciplinares y tres competencias Socioafectivas motivacionales: manejo de la interacción con el grupo, manifestación de valores y actitudes positivas hacia el estudiante y promoción de un vínculo entre el estudiante con su aprendizaje.

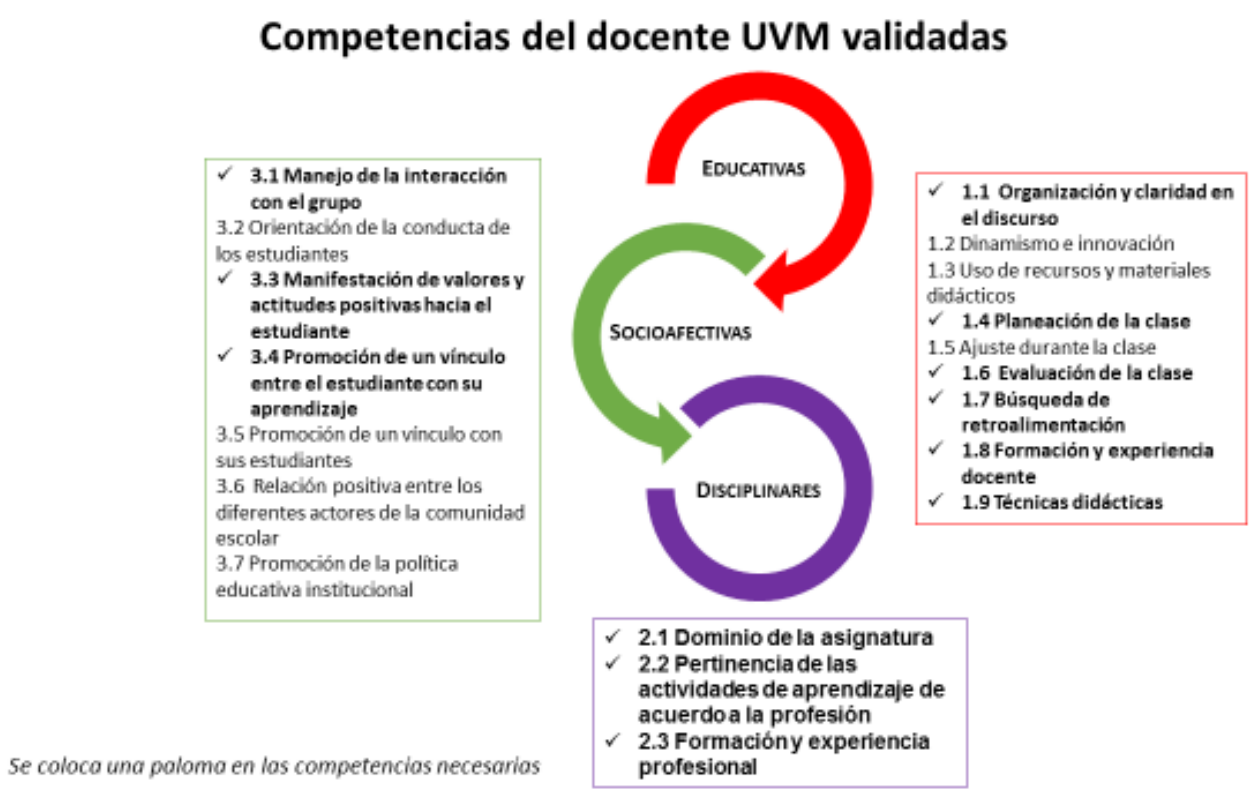

Figura 11. Se muestra el modelo de competencias docentes validado las figuras académicas. Se coloca una paloma en las competencias validadas como Necesarias.

\section{Discusión}

El objetivo del presente estudio fue describir el proceso de construcción del modelo de competencias docentes en la UVM. Proceso sistemático de revisión de la literatura y de adecuación empírica del modelo con comentarios tanto de docentes como de estudiantes de la universidad, validado por diferentes figuras académicas.

El modelo de desarrollo propuesto se inspiró en la propuesta de Flores-Hernández y cols. (2010) quienes desarrollaron su propuesta en una universidad pública mexicana acotada a docentes de medicina. Compuesto de cinco grupos de competencias: Disciplinar, Psicopedagógica, Humanística, Comunicación-evaluación y Solución de problemas.

En el caso de esta investigación el proceso inició con la revisión teórica y el apego a una propuesta preexistente, posteriormente se consolidó por medio de un instrumento aplicado a estudiantes y docentes. Este proceso puede ser útil para construir un camino sistemático que consolide un perfil docente en instituciones universitarias, acotado a las necesidades específicas de cada institución.

Así mismo, la validación es un punto fuerte del modelo, ya que los datos permitieron hacer una comparación entre diferentes áreas de conocimiento, encontrando que existe un perfil transversal o genérico del docente en UVM. 
Un punto clave para que este proceso pueda ser adoptado por otras instituciones, además de un procedimiento sistemático respaldado por un método mixto de investigación, es la incorporación de la perspectiva de diferentes actores involucrados, puesto que no sólo se recupera su voz, sino que al incluirla, en la implementación del modelo sus cimientos se comprenden mejor por la comunidad.

Una limitación importante fue el número de docentes que respondieron a la invitación de contestar el cuestionario y que representan sólo el $9 \%$ de los académicos de la universidad. Por otra parte, un aspecto que no se retomó en las mismas dimensiones que los docentes dentro de la validación fue la postura de los estudiantes, lo cual puede mejorarse teniendo un muestreo de estudiantes en diferentes ciclos de la formación para obtener datos diferentes o bien, que consoliden lo encontrado.

Un punto de desarrollo e investigación es la alineación de este modelo de competencias docentes con procesos de contratación, evaluación y formación profesional docente. A través de protocolos, guías de observación, manuales e instrumentos válidos y confiables, que enriquezcan tanto la labor como el alcance del perfil docente que se propone en el modelo de la Universidad.

\section{Conclusiones}

El modelo permite reconocer competencias básicas que cualquier docente en la Universidad despliega en sus aulas ya sea en una modalidad educativa dedicada al adulto trabajador estudiante a tiempo parcial o al estudiante de tiempo completo.

Las definiciones de cada grupo de competencias enriquece la caracterización de la práctica docente que se espera en las aulas de la universidad.

Un punto fuerte del modelo es que las competencias disciplinares son reconocidas por todas las figuras académicas involucradas en la validación como necesaria, aspecto que empata con la formación que se busca en esta Universidad.

\section{Referencias}

Academic Ranking of World Universities (2014). World Top 500 Universities. Shanghai: Shanghai Ranking Consultancy. http://www.shanghairanking.com/es/

Dolby, N. \& Litster, A. (2015). Understanding veterinarians as educators: an exploratory study. Teaching in Higher Education, 20 (3), 272-284. DOI: 10.1080/13562517.2014.1001835

Flores-Hernández, F., Martínez-González, A., Sánchez-Mendiola, M., García-Cabrero, B. y Reidl, L. (2010). Modelo de competencia docente del profesor de medicina en la UNAM. RELIEVE, 1-21.

Guzmán Ibarra, I. y Marín Uribe, R. (2011). La competencia y las competencias docentes: reflexiones sobre el concepto y la evaluación. Revista electrónica interuniversitaria de formación del profesorado, 36(14-1), 151-163.

Guzmán-Cedillo, YI. y Lima-Villeda, DN. (2014). Los rankings universitarios y las competencias docentes: un análisis de las mejores universidades. Memorias del VIII Congreso Internacional de Innovación Educativa del Instituto Politécnico Nacional, México. http://www.cgfie.ipn.mx/Paginas/Inicio.aspx

Hachfeld, A., Hahn, A., Scroeder, S., Anders, Y. \& Kunter, M. (2015). Should teachers be colorblind? How multicultural and egalitarian beliefs differentially relate to aspects of teachers' professional competence for teaching in diverse classrooms. Teaching and Teacher Education, 48, 44-55. http://dx.doi.org/10.1016/j.tate.2015.02.001

Mas Torelló, Ó. (2012). Las competencias del docente universitario: la percepción del alumno, de los expertos y del propio protagonista. REDU, 10(2), 299-318. http://red-u.net/redu/index.php/REDU/article/view/381 
Murzakhanovna, M., Meirbekovich, K., Erkinbekovich, Z., Pernebekovna, G., Koptleuovich, S. \& Kyrgyzbaevna, Z. (2013). The Problem of Using Competence-Based Approach and Information Technologies in Formation of Communicative Competence of the Future Specialists. Creative Education, 4 (8), 503-508. http://dx.doi.org/10.4236/ce.2013.48073

Pereyra, L. (2014). Evaluación docente. Manuscrito inédito, Dirección de Evaluación Educativa, Universidad del Valle de México. Distrito Federal, México.

Pereyra, L., Lima-Villeda, D.N. y Armenta, B. (2014). Escalas de evaluación y autoevaluación de competencias docentes en la Universidad del Valle de México. Memorias del Congreso Internacional de Educación 2014 de la Universidad Autónoma de Tlaxcala, México. http://posgradoeducacionuatx.org/congreso2015/?page_id=98

QSIntelligence Unit (2014). World University Rankings. https://www.iu.qs.com/university-rankings/world-universityrankings/

Reichheld, F. (2006) The Ultimate Question: Driving Good Profits and True Growth. EstadosUnidos: Harvard Business Review Press.

Rueda, M. (2006). Evaluación de la labor docente en el aula universitaria. México, UNAM.

Times Higher Education (2015). World University Rankings. https://www.timeshighereducation.co.uk/world-universityrankings/2015/world-ranking/\#/

Webel, C., \& Platt, D. (2015). The role of professional obligations in working to change one's teaching practices. Teaching and Teacher Education, 47, 204-217.

Witte, TC. \& Jansen, EP. (2015). In search of the excellent literature teacher. An inductive approach to constructing professional teaching standards. Teachers and Teaching: Theory and practice, 21(5), 565-583. http://dx.doi.org/10.1080/13540602.2014.995478 